Ann. Zootech., 1983, 32 (3), 383-396

\title{
Tolérance au climat tropical des taurillons Frisons et Créoles soumis à plusieurs régimes alimentaires. Détermination d'un indice climatique
}

\author{
P. BERBIGIER \\ I.N.R.A.-C.R.A.A.G., Station de Bioclimatologie, Domaine Duclos \\ 97170 Petit-Bourg, Guadeloupe.
}

\section{Résumé}

La thermotolérance de taurillons frisons et créoles, élevés sous abri, est étudiée en fonction du microclimat environnant et du régime alimentaire, à l'aide de trois critères : la température cutanée, la température rectale, le ryhme respiratoire.

Les taurillons frisons présentent des valeurs plus élevées de ces trois paramètres, surtout avec des régimes riches en énergie. Cependant, leur consommation et leur croissance restent supérieures à celles des créoles.

Un indice climatique commun aux deux génotypes est établi à partir de l'analyse en régression multiple de l'effet de la température ambiante, de la pression de vapeur, de la vitesse du vent, de la production de chaleur estimée et du poids des animaux sur la température rectale et le rythme respiratoire.

\section{Introduction}

Les races bovines à haute productivité des régions à climat tempéré sont en général incommodées par la chaleur lorsqu'elles sont introduites en climat tropical : ce phénomène s'accompagne souvent d'une réduction des performances, croissance ou production laitière. Il peut donc être utile d'apprécier la tolérance à un tel climat de différentes races ou croisements que l'on désire introduire sous les tropiques.

Ce travail a pour objet la comparaison de la thermotolérance d'une race bovine européenne à vocation laitière, la Française Frisonne Pie Noire (animaux frisons), à celle de la population bovine de Guadeloupe (animaux «créoles»), qui semble bien adaptée à la chaleur. Les critères de thermotolérance retenus sont la température cutanée, la température rectale et le rythme respiratoire. Ces deux derniers critères ont été fréquemment utilisés (Bianca \& Hales, 1970; AdEyemo et al., 1979 ; SeIf, Johnson \& LipPincotT, 1979 ; Morais, Espinosa \& SaAVEdra, 1979; Morais \& Espinosa, 1979 ; EsPinOSA, 1981 ; ANSEll, 1981). Cependant, la contrainte thermique du milieu est définie de manière plus précise que dans les travaux précédents, à partir non seulement du microclimat, mais aussi de paramètres caractéristiques de l'animal, de 
son poids et sa production de chaleur. De plus cette étude est effectuée en plein air, avec la seule protection d'un toit en tôle, et non en chambre climatique, comme beaucoup de travaux similaires.

\section{Matériel et méthodes}

\section{A. Animaux et alimentation}

10 taurillons frisons et 10 créoles de poids initiaux voisins (respectivement $262 \pm 25 \mathrm{~kg}$ et $234 \pm 19 \mathrm{~kg}$, moyenne \pm écart-type) mais d'âges différents (243 \pm 15 jours et $544 \pm 72$ jours) sont placés à l'attache dans une étable ouverte sur les côtés et munie d'un toit en tôle ondulée. Ce bâtiment, orienté perpendiculairement à la direction de l'alizé, est très bien ventilé. Les animaux reçoivent successivement, pendant des périodes de 2 mois, 3 régimes différents distribués ad libitum : un régime «luzerne » à base de luzerne déshydratée condensée, un régime «concentré » à base d'aliment concentré composé principalement de son de blé, un régime « ensilage » (ensilage de Pangola mélassé à 5 p. 100 , de teneur en matière sèche égale à $23,0 \pm 3,9$ p. 100 (moyenne \pm écart-type) réalisé à partir d'un fourrage vert dont la digestibilité in vitro estimée par la méthode de Tilley \& Terry, 1963 est égale à 50 p. 100). Les deux premiers régimes sont également complétés par le même ensilage, distribué à raison de 2 p. 100 du poids vif des animaux en matière fraîche. La séquence des trois régimes est répétée deux fois pour prendre en compte les variations climatiques saisonnières, l'expérience durant un an. Les quantités d'aliment ingérées au cours des 10 premiers jours (période d'adaptation) de chaque période expérimentale ne sont pas prises en compte.

Le poids des animaux est déterminé, par double pesée, 9 et 10 jours après le début, ainsi qu'à la fin de chaque période expérimentale.

Un animal de chaque race a été éliminé en cours d'expérience pour des raisons indépendantes du climat.

\section{B. Mesures bioclimatiques}

Elles sont effectuées deux fois par semaine, chaque fois sur la moitié des animaux de chaque génotype. Elles sont répétées à deux périodes de la journée, de $10 \mathrm{~h} 30$ à 12 heures et de $14 \mathrm{~h} 30$ à 16 heures, sur les mêmes animaux. Les résultats n'étant pas statistiquement différents pour les 2 périodes, seules les moyennes journalières relatives à chaque animal secont considérées.

Les paramètres mesurés sont les suivants :

- Ia température cutanée mesurée à la sonde de contact (moyenne des deux épaules, flancs et cuisses),

- la température rectale,

- le rythme respiratoire, par comptage des battements de flanc.

Simultanément, les paramètres du microclimat (température du thermomètre sec Ta, température du thermomètre humide $T h$, vitesse du vent v) sont mesurés au niveau des taurillons. La pression de vapeur $\mathrm{Pa}$ est calculée à partir de Ta et Th. Un thermographe permet d'enregistrer les températures maximales et minimales. 
Les mesures bioclimatiques ne sont pas effectuées pendant les dix premiers jours de chaque période expérimentale.

\section{Estimation de la production de chaleur} fixée.

Elle est estimée par différence entre l'énergie métabolisable ingérée et l'énergie

La teneur en énergie métabolisable (EM) de chaque ration est estimée à partir de la teneur en EM de chacun des aliments constitutifs. Pour l'aliment concentré et la luzerne condensée, les valeurs retenues sont tirées des tables établies par l'I.N.R.A. (I.N.R.A., 1978). Pour l'ensilage, la teneur en EM est estimée à partir de l'analyse du fourrage vert utilisé et de la teneur en EM de la mélasse de canne à sucre (I.N.R.A., 1978). Les quantités de matière sèche ingérée en ensilage dans le cas des régimes «concentré 》 et «luzerne» représentant 20 p. 100 environ des quantités totales de matière sèche ingérée, il n'est pas tenu compte des phénomènes d’associativité (I.N.R.A., 1978).

L'énergie fixée est estimée à partir de la dissection de taurillons frisons et créoles. Le poids des dépôts adipeux totaux $(\mathrm{Y}, \mathrm{kg})$ est mesuré sur une population de 11 animaux de chaque génotype (XANDE, non publié) ; son évolution en fonction du poids vif vide (PVV, $\mathrm{kg}$ ) est décrite par les équations suivantes :

$$
\begin{aligned}
& \mathrm{Y}=0,001379 \mathrm{PVV}^{1,77} \text { pour les taurillons frisons, } \\
& \mathrm{Y}=0,002448 \mathrm{PVV}^{1,69} \text { pour les taurillons créoles. }
\end{aligned}
$$

On peut en déduire, selon la méthode décrite par Robelin \& GeAY (1978), la relation d'allométrie entre le poids vif vide et la valeur calorifique du corps entier ( $E$, Mcal), qui est de la forme : $\mathrm{E}=\mathrm{a}(\mathrm{PVV})^{\mathrm{b}}$, ainsi que la valeur calorifique du croît à partir de la dérivée de cette équation $\frac{\mathrm{dE}}{\mathrm{d}(\mathrm{PVV})}=\mathrm{a} \cdot \mathrm{b} \cdot(\mathrm{PVV})^{\mathrm{l},-1}$. D'autre part, le rapport du poids vif vide au poids vif des animaux est trouvé égal à 0,833 pour les frisons et à 0.866 pour les créoles, valeurs peu dépendantes de la ration.

A partir de ces données, il est possible d'obtenir l'expression de l'énergie fixée en fonction du poids vif et du gain de poids vif. Pour les animaux disséqués (frisons : GMQ $=1,06 \mathrm{~kg}$, créoles : GMQ $=0,72 \mathrm{~kg}$ ), celle-ci s'écrit :

$$
\begin{aligned}
& \text { frisons: }: \mathrm{EF}=\mathrm{GMQ} \times\left(1,081+0,01705 \times \mathbf{P}^{0,76}\right) \\
& \text { créoles }: \mathrm{EF}=\mathrm{GMQ} \times\left(1,150+0,03236 \times \mathrm{P}^{0,68}\right)
\end{aligned}
$$

où EF est l'énergie fixée (Mcal/jour), GMQ le gain du poids moyen quotidien ( $\mathrm{kg} / \mathrm{jour}$ ) et $\mathrm{P}$ le poids vif $(\mathrm{kg})$.

L'énergie fixée étant proportionnelle au gain de poids moyen élevé à la puissance 1,42 (Robel.IN, communication personnelle; I.N.R.A., 1978), il convient de multiplier les expressions précédentes par un coefficient correctif $\left(\frac{\text { GMQ ex }}{\text { GMQ diss }}\right)^{1,42}$ où GMQ ex est le gain des animaux expérimentaux et GMQ diss celui des animaux disséqués, pour obtenir l'énergie fixée des premiers. 
Dans le cas du régime «ensilage» avec lequel les animaux sont au-dessous de l'entretien, la production de chaleur est calculée à partir de l'énergie métabolisable ingérée et de son rendement pour l'entretien, $k_{m}$, pris égal à 0,67 (I.N.R.A., 1978).

Le rendement peut être considéré comme le rapport : $k_{m}=\frac{E M_{I}-E C}{E_{I}}$, où $E_{I}$ est l'énergie métabolisable ingérée et EC l'extra-chaleur. A partir de cette expression, et en remarquant que la production de chaleur $\mathrm{Q}$ est égale à la somme du métabolisme $\mathrm{au}$ jeûne $\mathrm{MJ}$ et de l'extra-chaleur $\mathrm{EC}$, il est possible d'écrire :

$$
\mathrm{Q}=\left(1-\mathrm{k}_{\mathrm{rn}}\right) \mathrm{EM}_{\mathrm{I}}+\mathbf{M J}=\left(1-\mathrm{k}_{\mathrm{m}}\right) \mathrm{EM}_{\mathrm{I}}+\mathrm{k}_{\mathrm{m}} \mathbf{E M}_{\mathrm{E}}
$$

où $\mathrm{EM}_{\mathrm{E}}$ est l'énergie métabolisable d'entretien, estimée à $110 \mathrm{kcal} / \mathrm{kg}^{0,75} \times$ jour (I.N.R.A., 1978). I1 faut noter qu'au-dessous de l'entretien, $Q$ est toujours supérieure à $\mathbf{E M}_{\mathrm{I}}$, et qu'une partie de la chaleur dissipée est puisée dans les réserves corporelles (GMQ négatifs).

La production de chaleur totale est ramenée à l'unité de surface cutanée en utilisant l'expression suivante (WEBSTER, 1974) :

$$
\mathrm{E}=0,09 \times \mathbf{P}^{0,67}
$$

où $\mathbf{S}$ est la surface cutanée $\left(\mathrm{m}^{2}\right)$ et le $\mathbf{P}$ le poids $(\mathrm{kg})$.

\section{Analyse statistique}

A l'intérieur d'un génotype, la liaison entre les moyennes journalières des températures cutanée, rectale, du rythme respiratoire et les variables climatiques, la production de chaleur et le poids est analysée par régression multiple linéaire. Pour ces deux dernières variables, la valeur affectée à une journée expérimentale est la valeur moyenne de la période expérimentale correspondante. Les résultats ainsi obtenus pour chaque demi-lot d'animaux n'étant pas différents, toutes les journées d'expérience sont traitées ensemble.

\section{Résultats}

Les valeurs moyennes relatives à chaque régime des quantités de matière sèche ingérée, du gain de poids, de la production de chaleur, des températures corporelles et du rythme respiratoire sont résumées sur le tableau 1.

\section{A. Effet de la race sur les températures corporelles et le rythme respiratoire}

Les moyennes relatives à chaque animal calculées sur toutes les journées d'expérience, comparées par test $t$, montrent une différence significative entre les deux races : pour les créoles et les frisons, les températures de surface (Ts) sont respectivement de 34,98 et $35,59^{\circ} \mathrm{C}(\mathrm{P}<0,001)$, les températures rectales $(\mathrm{Tr})$ de 38,68 et $38,95^{\circ} \mathrm{C}$ $(\mathrm{P}<0,001)$, et les rythmes respiratoires ( $\mathrm{Rr})$ de 27,0 et 48,5 respirations/minute $(\mathrm{P}<0,001)$.

Les différences entre races sont d'autant plus accentuées que la température ambiante est plus élevée et la production de chaleur plus grande. 


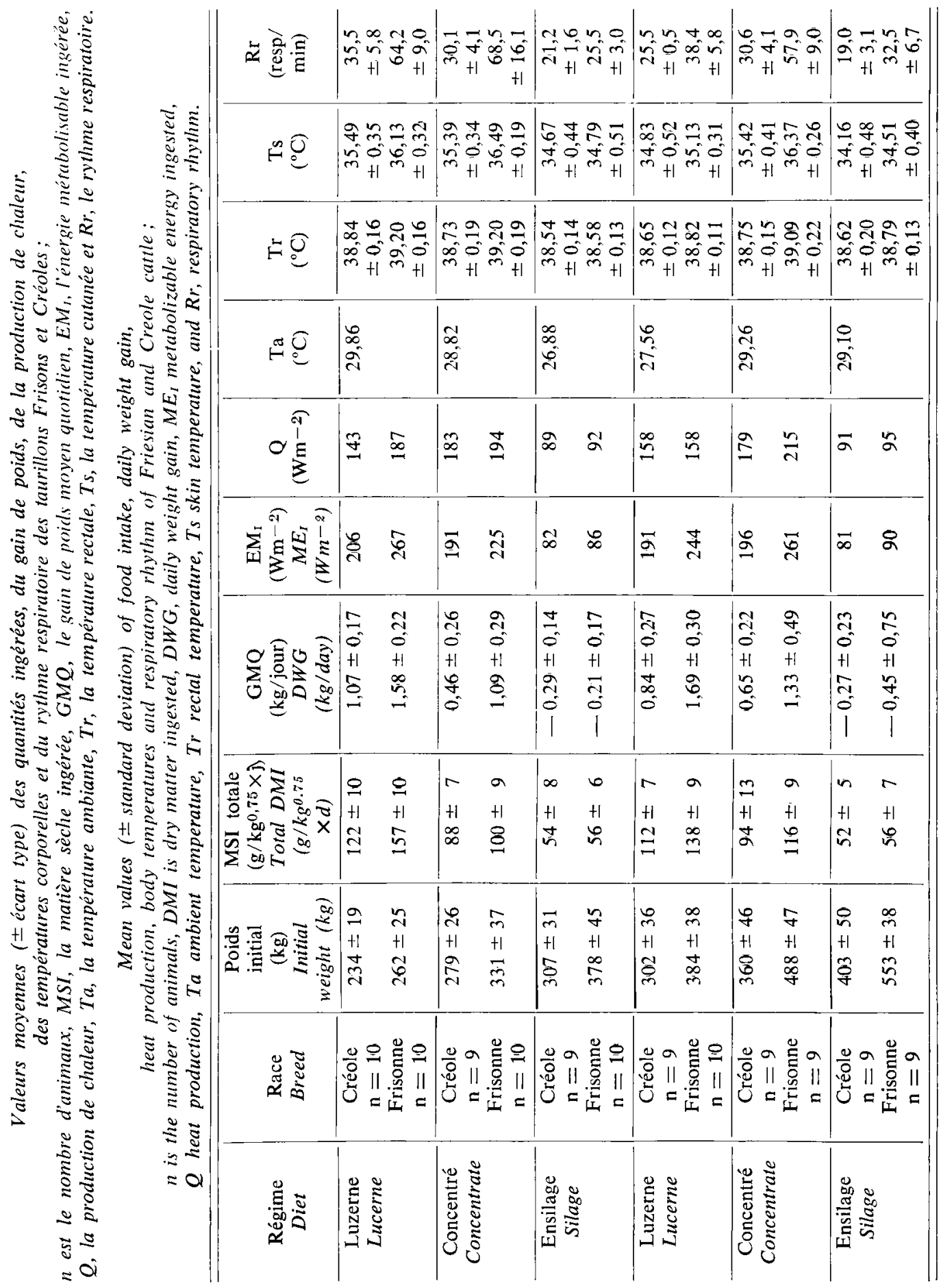


Par exemple, dans le cas de la première période de distribution du régime "concentré », où la température ambiante est de $28,82^{\circ} \mathrm{C}$, les différences entre les frisons et les créoles sont respectivement de $1,10^{\circ} \mathrm{C}, 0,51^{\circ} \mathrm{C}$ et 40,5 respirations/minute, pour $\mathrm{Ts}$, $\operatorname{Tr}$ et $\operatorname{Rr}(\mathrm{P}<0,001$ dans tous les cas). Il convient de remarquer que la production de chaleur des frisons est alors supérieure de 6 p. $100\left(201 \mathrm{Wm}^{-2}\right.$ contre $190 \mathrm{Wm}^{-2}$ ) à celle des créoles. En revanche, pour la première période de distribution du régime "ensilage», où la température ambiante est de $26,88^{\circ} \mathrm{C}$, entre les frisons et les créoles les différences de température cutanée $\left(0,33^{\circ} \mathrm{C}\right)$ et de température rectale $\left(0,04{ }^{\circ} \mathrm{C}\right)$ ne sont pas significatives mais la différence de rythme respiratoire $(4,7$ respirations $/ \mathrm{mn})$ est significative au seuil 1 p. 100.

\section{B. Effets du microclimat, du poids et de la production de chaleur}

Les résultats portent sur 65 journées de mesures. La moyenne sur ces journées des paramètres du microclimat pris au moment des mesures est :

- température ambiante : $\mathrm{Ta}=28,5 \pm 1,6^{\circ} \mathrm{C}$ (moyenne \pm écart-type) ;

- pression de vapeur : $\mathrm{Pa}=2650 \pm 330$ Pascal ;

- vitesse du vent : $\mathrm{v}=2,20 \pm 0,86 \mathrm{~ms}^{-1}$.

La moyenne des températures minimales des nuits précédant les jours de mesure est :

$$
\operatorname{Tmin}=23,8 \pm 2,0^{\circ} \mathrm{C} \text {. }
$$

Les équations de régression multiple obtenues sont présentées sur le tableau 2. La température minimale de la nuit précédente, dont l'effet (test $F$ ) n'atteint le seuil de signification qu'en une occasion, n'est pas prise en compte, non plus que la température maximale du jour, qui est pratiquement égale à la température $\mathrm{Ta}$ au moment de la mesure. La production de chaleur a un effet significatif $(P<0,001)$ sur les 6 variables expliquées ainsi que la température ambiante $(P<0,001$ dans 4 cas, $\mathbf{P}<0,01$ dans 1 cas, $\mathbf{P}<0,05$ dans 1 cas) ; celui du poids est significatif 5 fois sur 6 , celui de la pression de vapeur 4 fois sur 6 et celui du vent 3 fois sur 6 . Cet effet est toujours positif dans le cas de la production de chaleur et de la température ambiante, toujours négatif dans le cas du vent et du poids vif. Dans le cas de la pression de vapeur, il est positif, pour les deux génotypes, sur la température rectale et le rythme respiratoire et négatif sur la température cutanée.

Pour chaque variable expliquée, les coefficients de régression relatifs à une variable explicative donnée sont en général plus élevés en valeur absolue pour les taurillons frisons que pour les créoles, sauf dans le cas du poids vif, où l'inverse se produit. Cependant, dans ce dernier cas, les coefficients, comparés deux à deux par test $t$, ne sont jamais significativement différents. De plus, pour la température cutanée, les coefficients de régression relatifs aux trois variables climatiques sont remarquablement identiques pour les deux génotypes, alors que ceux relatifs au poids, bien que non significativement différents, sont très dispersés et que ceux relatifs à la production de chaleur sont significativement différents $(\mathrm{P}<0,01)$.

\section{Etablissement d'un indice climatique}

Pour comparer la thermotolérance des deux génotypes, il est nécessaire d'avoir une référence commune caractérisant l'interaction animal-milieu ambiant. Or, dans un 


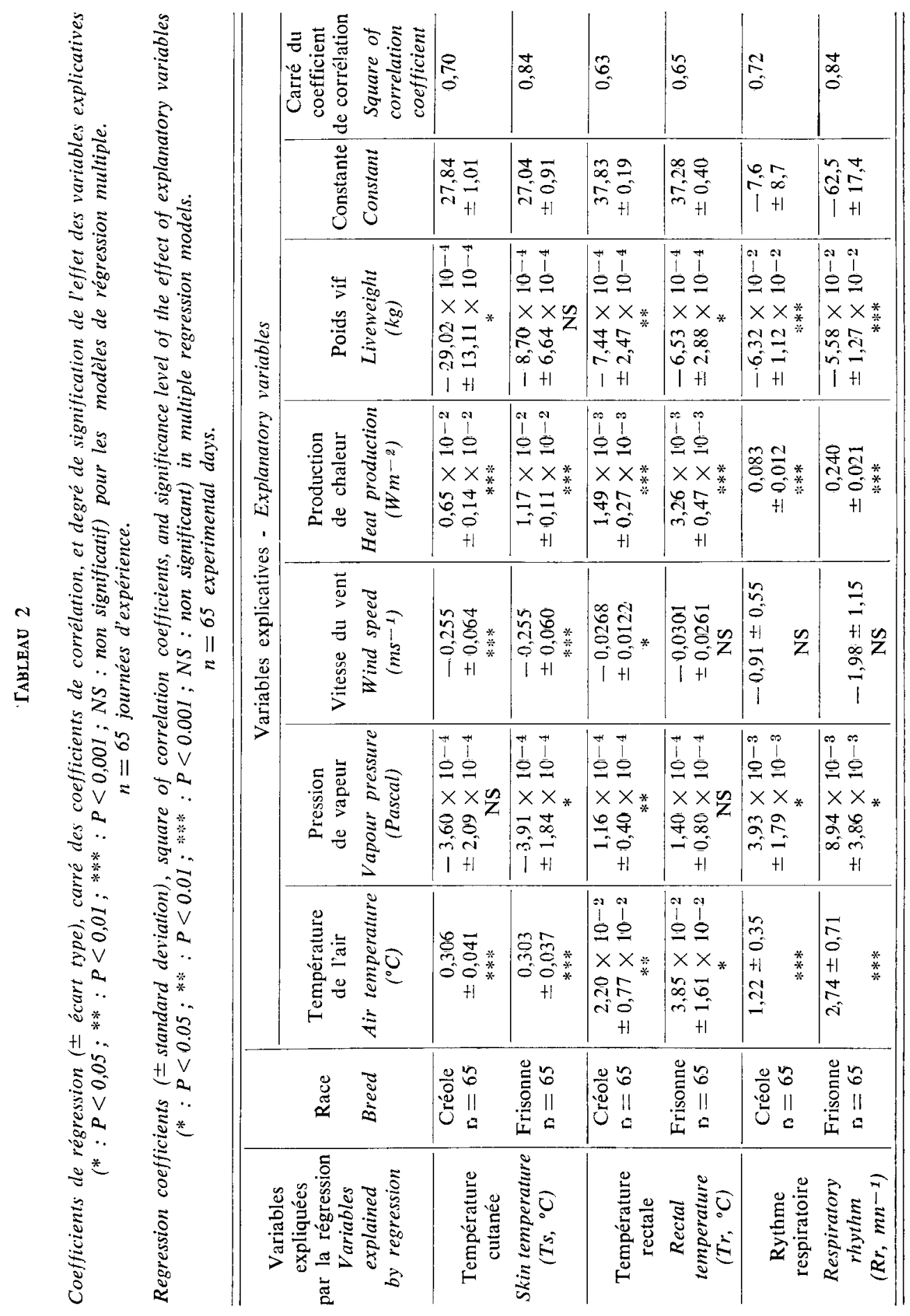


milieu non contrôlé, plusieurs paramètres climatiques agissent simultanément; de plus leur action dépend des caractéristiques physiques de l'animal et de sa production de chaleur (BERBigier, 1978). L'action globale du climat peut être décrite par une fonction de ces différents paramètres, fonction qui, lorsqu'ils varient dans des limites suffisamment étroites, peut être ramenée à une simple combinaison linéaire de ceux-ci. L'obtention d'un indice commun aux deux génotypes nécessite alors une proportionnalité des effets dus à chacune des variables explicatives.

Une telle combinaison linéaire commune aux deux génotypes ne peut être obtenue à partir des modèles relatifs à la température cutanée : en effet, si les coefficients de régression relatifs aux variables climatiques sont pratiquement égaux deux à deux, ceux relatifs au poids vif et surtout à la production de chaleur sont différents (tabl. 2).

En revanche, il est possible d'obtenir une telle référence à partir des équations relatives à la température rectale et au rythme respiratoire. Si nous divisons, dans chacune des quatre équations de régression multiple relatives à ces deux variables et aux deux génotypes, tous les coefficients de régression et leurs écarts-types par le coefficient de régression relatif à la température de l'air Ta, nous obtenons quatre expressions (que nous appellerons "réduites》) où le coefficient de régression "réduit 》 de Ta est égal à 1. Les coefficients de régression «réduits», affectés de leurs écarts-types «réduits», relatifs à la même variable explicative dans les quatre équations, ne sont plus statistiquement différents, à l'exception des coefficients relatifs au poids. Avec cette restriction, ceci montre donc que les deux génotypes réagissent, avec des intensités différentes, à une même combinaison linéaire des variables explicatives, qu'il s'agisse de la température rectale ou du rythme respiratoire. Une approximation de cette combinaison linéaire peut être obtenue en calculant la moyenne des quatre coefficients de régression « réduits» relatifs à chaque variable explicative (tabl. 3).

Pour améliorer la forme de l'indice climatique ainsi obtenu, faisons rentrer dans son expression, pour toutes les variables à l'exception de $\mathrm{Ta}$, les écarts de celles-ci à une valeur caractéristique. Si nous choisissons, pour les variables climatiques la moyenne des valeurs obtenues pendant les journées de mesure, pour la production de chaleur : $100 \mathrm{Wm}^{-2}$, qui est une valeur simple et proche du métabolisme d'entretien (tabl. 1), pour le poids : $300 \mathrm{~kg}$, l'indice climatique s'écrit ainsi :

$$
\begin{gathered}
\mathrm{I}=\mathrm{Ta}+3,85 \times 10^{-3}(\mathrm{~Pa}-2650)-0,868(\mathrm{v}-2,20)+7,71 \times 10^{-2}(\mathrm{Q}-100) \\
\quad-3,07 \times 10^{-2}(\mathrm{P}-300)
\end{gathered}
$$

où Ta $\left({ }^{\circ} \mathrm{C}\right)$ est la température de l'air, $\mathrm{Pa}$ (Pascal) la pression de vapeur, $\mathrm{v}\left(\mathrm{ms}^{-1}\right)$ la vitesse du vent, $\mathbf{Q}\left(\mathrm{Wm}^{-2}\right)$ la production de chaleur et $\mathbf{P}(\mathrm{kg})$ le poids vif des taurillons. Il s'exprime en ${ }^{\circ} \mathrm{C}$ et représente l'effet, sur la température rectale et le rythme respiratoire, de la température de l'air, corrigé par celui de l'écart à leur valeur caractéristique des autres variables.

L'indice I peut être considéré comme une «température équivalente» (MoNTEITH, 1973). Il est théoriquement égal à la température ambiante qui, pour un animal de $300 \mathrm{~kg}$ ayant une production de chaleur par unité de surface de peau de $100 \mathrm{Wm}^{-2}$, placé dans une ambiance à pression de valeur et à ventilation constantes égales respectivement à $2650 \mathrm{~Pa}$ et à $2,2 \mathrm{~ms}^{-1}$, aurait sur la température rectale et le rythme respiratoire le même effet que la combinaison des valeurs des variables explicatives obtenue en milieu non contrôlé.

Lorsque la pression de vapeur augmente de $100 \mathrm{~Pa}$ (ce qui correspond à une augmentation de 2,6 p. 100 de l'humidité relative), I augmente de $0,4^{\circ} \mathrm{C}$; lorsque 


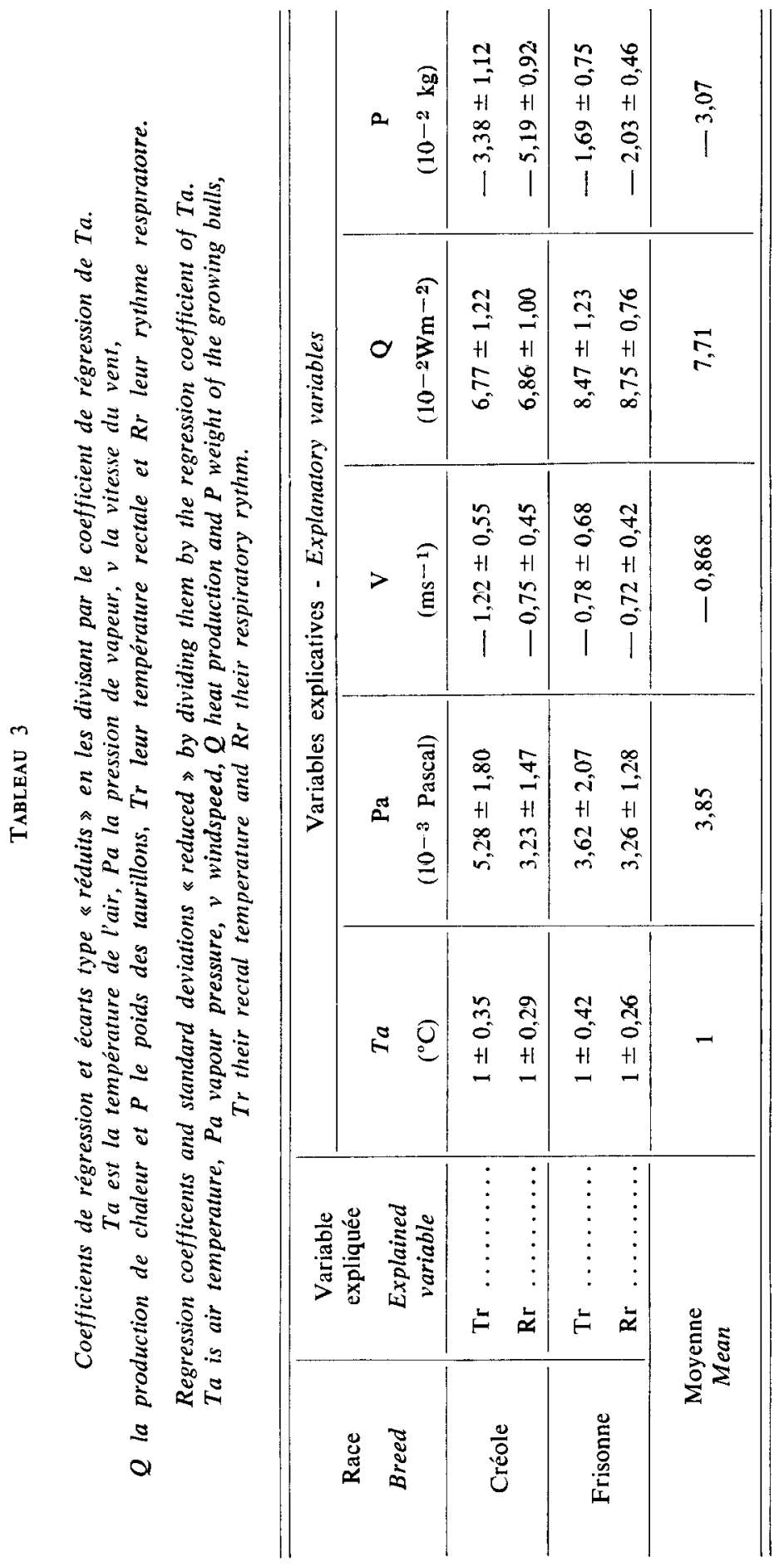


le vent augmente de $1 \mathrm{~ms}^{-1}$, I diminue de $0,9^{\circ} \mathrm{C}$ : lorsque la production de chaleur augmente de $100 \mathrm{Wm}^{-2}$ (ce qui représente, pour les taurillons frisons, la différence entre le régime "concentré » et le régime «ensilage»), I présente une augmentation très importante de $7,7^{\circ} \mathrm{C}$, du même ordre que l'écart maximum entre les températures ambiantes prises au moment des mesures; enfin, lorsque le poids augmente de $100 \mathrm{~kg}$, I diminue de $3,1^{\circ} \mathrm{C}$.

\section{Effet de lindice I sur $\operatorname{Tr}$ et $\mathrm{Rr}$}

Les relations obtenues entre la température rectale et le rythme respiratoire d'une part, l'indice I d'autre part, pour les deux génotypes, sont portées sur la figure 1. Dans les deux cas, la droite de régression obtenue pour les taurillons frisons se situe au-dessus de celle obtenue pour les créoles. Les équations de régression correspondantes sont :

$$
\begin{aligned}
& \text { FFPN } \\
& \begin{array}{rlr}
: \mathrm{Tr} & =0,0369 \times \mathbf{I}+37,84 & \mathbf{R}^{2}=0,59 \\
\mathrm{Rr} & =2,73 \times \mathbf{I}-33,12 & \mathbf{R}^{2}=0,82
\end{array} \\
& \text { : } \operatorname{Tr}=0,0222 \times \mathrm{I}+37,99 \quad \mathrm{R}^{2}=0,50 \\
& \mathrm{Rr}=1,20 \quad \times \mathrm{I}-10,34 \quad \mathrm{R}^{2}=0,67
\end{aligned}
$$

Où $\mathrm{Tr}$ et $\mathrm{I}$ sont exprimés en ${ }^{\circ} \mathrm{C}, \mathrm{Rr}$ en respirations/minute. Aussi bien pour $\operatorname{Tr}$ que $\mathrm{Rr}$, les pentes des droites de régression des FFPN sont significativement plus fortes $(\mathrm{P}<0,001)$ que celles des créoles. Pour les plus basses valeurs de I observées, les deux droites tendant à se rejoindre.

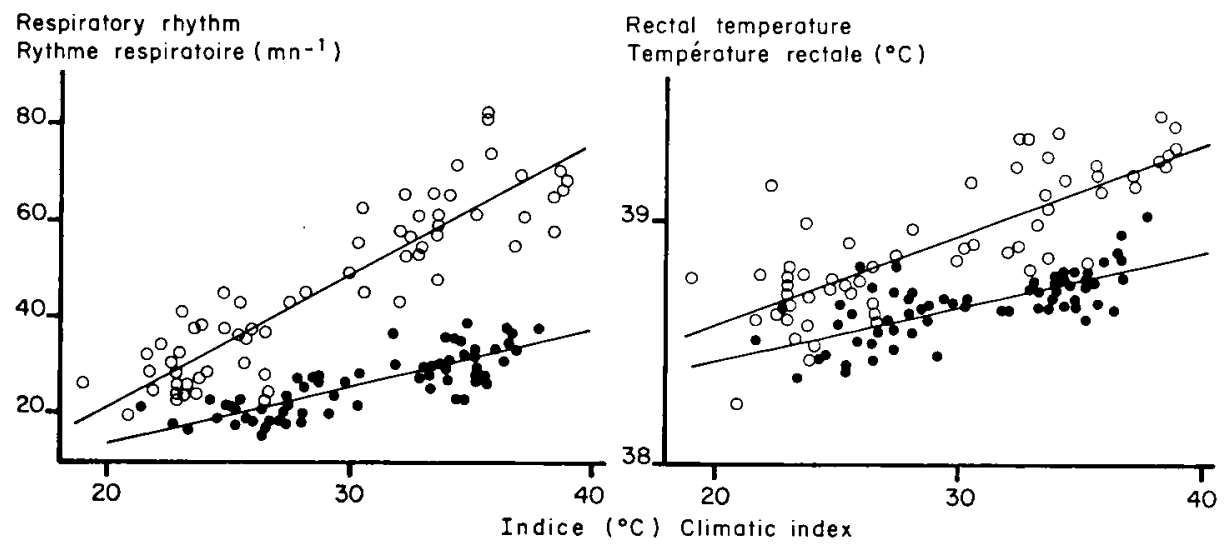

FIG. 1

Relations entre la température rectale, le rythme respiratoire et l'indice climatique I chez les taurillons frisons et créoles.

Interrelationships between rectal temperature, respiratory rhythm and climatic index 1 for Friesian and Creole growing bulls.

Frisons.

- Créoles. 


\section{Discussion}

Le résultat le plus important est l'effet du génotype sur la thermotolérance des taurillons. Celui-ci s'observe aussi bien sur les moyennes tous régimes confondus que sur les valeurs journalières des températures rectale et cutanée et du rythme respiratoire. Les taurillons frisons ont plus de mal que les créoles à équilibrer leurs échanges de chaleur, ce qui se traduit par des niveaux plus élevés de ces trois variables. Toutefois, cet écart à tendance à se réduire lorsque la contrainte thermique diminue, soit par une réduction de la production de chaleur, soit par un rafraîchissement du microclimat. Pour les régimes à haute teneur en énergie, la différence entre génotypes est certainement due en partie aux quantités d'aliments ingérées plus importantes, donc à la production de chaleur plus forte des taurillons frisons. Cependant, la comparaison des deux génotypes à l'aide de l'indice I, dans lequel l'effet de la production de chaleur est pris en compte, montre que, toutes choses étant égales par ailleurs, il subsiste une différence de thermotolérance entre les taurillons frisons et créoles. Malgré cela, les frisons ont une consommation et une vitesse de croissance supérieures à celles des créoles, du moins dans le cas des deux régimes les plus énergétiques. Il est probable qu'avec ceux-ci, le potentiel de croissance des taurillons frisons peut s'exprimer, alors qu'avec l'ensilage de Pangola, peu digestible, la capacité du rumen constitue une limite de l'ingestion et de la vitesse de croissance. Le climat, même dans l'hypothèse où il freine la croissance des frisons, n'a pas un effet suffisant pour la ramener au niveau de celle des créoles. Cependant, la conception de l'expérience (régimes successifs entraînant des phénomènes de rattrapage) ne nous permet pas d'analyser plus en détail l'action du climat sur la consommation et la vitesse de croissance.

La construction de l'indice I est basée sur la proportionnalité des effets des variables explicatives. Entre la température rectale et le rythme respiratoire, celle-ci se comprend aisément, car ces deux paramètres sont étroitement liés (INGRAM \& MounT, 1975). Dans l'expérience présente, les coefficients de détermination entre $\mathrm{Tr}$ et $\mathrm{Rr}$ sont respectivement $\mathrm{R}^{2}=0,51$ et $\mathrm{R}^{2}=0,72$ pour les créoles et les FFPN (liaison significative à $\mathrm{P}<0,001$ dans les deux cas). Entre les génotypes, cette proportionnalité montre que le poids de chacune des voies d'échange thermique dans le bilan thermique total est du même ordre chez les taurillons frisons et créoles.

La température cutanée réagit d'une manière différente à ces mêmes variables : en effet, d'une part, elle ne fait pas l'objet d'une thermorégulation aussi rigoureuse que $\mathrm{Tr}$, et d'autre part, étant caractéristique d'une zone au contact direct de l'ambiance, elle est plus étroitement liée au climat que $\mathrm{Tr}$ et $\mathrm{Rr}$.

L'action négative de la pression de vapeur sur Ts est difficilement explicable car lorsque l'humidité ambiante augmente, la thermolyse latente devrait être réduite, ce qui devrait provoquer une augmentation de la température cutanée : cependant, cet effet est à la limite de la signification.

Le rôle du poids est plus difficile à expliquer. En premier lieu, son rôle et celui de l'âge sont indissociables statistiquement. Cependant, aucun auteur ne mentionne un effet de l'âge sur $\mathrm{Tr}$ et $\mathrm{Rr}$ chez les taurillons à ce stade de développement.

L'action négative du poids sur $\operatorname{Tr}$ et $\mathrm{Rr}$ est inattendue, car en général les gros animaux dissipent moins bien la chaleur que les petits (Monteith, 1973). Cependant, en milieu de journée, les taurillons sont en situation non de dissipation, mais de stockage 
de chaleur : plus les animaux sont lourds, plus le rapport surface/poids est faible, et plus la chaleur stockée peut être répartie dans une masse importante, d'où une moindre augmentation de $\operatorname{Tr}$ (KLElBER, 1962). Il s'agit probablement ici, non de stress thermique, mais des fluctuations nycthémérales de $\operatorname{Tr}$, qui sont atténuées chez les gros animaux.

L'effet plus accentué du poids chez les créoles que chez les frisons est peut-être dû aux différences de morphologie ou à la différence de poids entre les deux génotypes. Quelle qu'en soit l'origine, cette différence est gênante car elle rend approximative la comparaison des réactions à la chaleur des deux génotypes à l'aide de l'indice $I$. Cependant, dans le cas présent, l'écart est tel qu'un indice moyen est suffisant.

L'indice I pose d'autres problèmes. D'une part, il a été établi sous un abri en tôle. Même si celui-ci est bien ventilé, de sorte que la température du corps noir ne dépasse la température de l'air que de $1,5^{\circ} \mathrm{C}$ au maximum, le rayonnement infrarouge du toit n'est pas pris en compte explicitement dans l'indice. D'autre part, il incorpore des valeurs instantanées des variables climatiques et des moyennes sur six semaines de la production de chaleur. Tel qu'il est, cependant, il semble bien représenter la contrainte thermique dans les conditions de l'expérience.

La plupart des indices climatiques existants ne prennent en compte que la température et l'humidité. Si nous considérons comme constants la vitesse du vent, la production de chaleur et le poids, et si nous convertissons la pression de vapeur en température de point de rosée $(\mathrm{Td})$, les variations de $\mathrm{Ta}$ et $\mathrm{Td}$ interviennent respectivement pour 62 p. 100 et 38 p. 100 dans la variation globale de l'indice I. Pour le THI (Temperature-Humidity Index) (JoHnson, 1980), ces proportions sont de 74 et 26 p. 100 et pour l'indice de BIANCA (1962), de 49 et 52 p. 100. Les résultats présents se situent donc dans une position intermédiaire par rapport à ces deux indices.

\section{Conclusion}

De nombreux indices climatiques ont été élaborés auparavant (cf. la revue bibliographique de STARr, 1981). Le plus connu reste le THI (Temperature-Humidity Index), établi pour les vaches laitières (Johnson, 1980). Par rapport à eux, l'originalité de l'indice présenté ici est, d'une part d'avoir été établi en conditions naturelles, d'autre part de prendre en compte la production de chaleur, qui est, nous lavons vu, un terme essentiel de la contrainte thermique.

Nous avons démontré la meilleure résistance à la chaleur des taurillons créoles par rapport aux FFPN ; cependant, dans les conditions expérimentales, les performances de croissance de ces derniers restent encore supérieures. Nous avons été amenés à développer un indice climatique original, tenant compte de la production de chaleur des animaux, qui, une fois amélioré pour intégrer l'effet du rayonnement solaire et de la pluie, pourra servir à caractériser l'action du climat tropical sur les bovins.

Accepté pour publication en avril 1983.

\section{Remerciements}

L'auteur tient à remercier M. Armand Grude, Directeur du domaine de Gardel pour son aide matérielle efficace. 


\section{Summary \\ Resistance to tropical climate of Friesian and Creole growing bulls fed different diets. Determination of a climatic index}

The thermotolerance of FFPPN and Creole growing bulls raised under shelter is studied according to microclimate and type of feed using three criteria : skin temperature, rectal temperature and respiratory rhythm.

For Friesian bulls, the values of these parameters were generally higher, particularly for the diets with the highest energy content. However, their food intake and live weight gain was better than those of Creole bulls.

From multiple regression analysis of the effect of air temperature, vapour pressure, windspeed, estimated heat production and weight of the animals on rectal temperature and respiratory rhythm (tabl. 2) a common climatic index for both genotypes was established (tabl. 3). This index fitted for uncontrolled environment takes into account animal characteristics, such as heat production and weight.

\section{Références bibliographiques}

Adeyemo O., Heath E., Adavevoh B.K., Steinbach J., Olaloku E.A., 1979. Some physiological and behavioural responses in Bos Indicus and Bos Taurus heifers acclimatized to the hot humid seasonal equatorial climate. Int. J. Biometeor., 23 (3), 231-241.

ANSELL R.H., 1981. Extreme heat stress in dairy cattle and its alleviation : a case report, 285-306. In : Clark J.A., Environmental aspects of housing for animal production. Butterworths, London.

Berbigier P., 1978. Echanges thermiques des animaux d'élevage en fonction des paramètres climatiques. B.T.I., 328-329, 129-151.

BIANCA W., 1962. Relative importance of dry- and wet-bulb temperature in causing heat stress in cattle. Nature, 195, 251-252.

Bianca W., Hales J.R.S., 1970. Sweating, panting and body temperature of new-born and one-year old calves at high environmental temperature. Br. Vet. J., 126, 45-53.

EsPinOSA J., 1981. Influencia de temperaturas y higrometrías elevadas sobre la temperatura corporal y la frecuencia respiratoria en el ternero. Revista de Salud Animal, 3 (1), 133-147.

I.N.R.A., 1978. Alimentation des ruminants, 47-88. I.N.R.A. Publications, Versailles.

INGRAM D.L., MOUNT L.E., 1975. Man and animals in hot environments, 183 p. SpringerVerlag, New York.

JoHnson H.D., 1980. Environmental management of cattle to minimize the stress of climatic change. Biometeorology, 7 (2), suplement to Int. J. Biometeor., 24, 65-78.

KLeIBer M., 1962. The fire of life, 454 p. John Wiley and Sons, New York.

Montelth J.L., 1973. Principes of environmental physics, 241 p. Edward Arnold, London.

Morais M., Espinosa J., 1979. Efecto de la temperatura ambiental y la humedad relativa sobre la temperatura rectal y la frecuencia respiratoria en vacas Cebù no lactantes. Revista de Salud Animal, I (1), 83-88.

Morais M., Espinosa J., SaAvedra L., 1979. Influencia de la temperatura ambiental y la humedad relativa sobre la temperatura rectal y la frecuencia respiratoria en vacas Holstein y de los cruces 3/4-1/4 y 5/8-3/8 $\mathrm{H} \times \mathrm{C}$ en condiciones cálidohúmedas. Revista de Salud Animal, I (3), 63-77. 
Robelin J., Geay Y., 1978. Estimation de la composition chimique du corps entier des bovins à partir du poids des clépôts adipeux totaux. Ann. Zootech., 27, 159-167.

Seif S.M., Johnson H.D., Lippincort A.C., 1979. The effect of heat exposure $\left(31^{\circ} \mathrm{C}\right)$ on Zebu and Scottish Highland cattle. Int. J. Biometeor., 23 (1), 9-14.

Starr J.R., 1981. Climate and the need for housing. 19-35. In : Clark J.A., Environmental aspects of housing for animal production, Butterworths, London.

Tilley J.M.A., Terry R.A., 1963. A two stage technique for the in vitro digestion of forage crops. J. Br. Grasst. Soc., 18 (2), 104-110.

Webster A.J.F., 1974. Heat loss from cattle with particular emphasis on the effects of cold, 205-231. In : MonTHEith J.L., MOUNT L.E., Heat loss from animals and man, Butterworths, London. 